\title{
Electrically tunable hole tunnelling from a single self-assembled quantum dot embedded in an $n$-i-Schottky photovoltaic cell
}

Cite as: Appl. Phys. Lett. 99, 031102 (2011); https://doi.org/10.1063/1.3614418

Submitted: 10 June 2011 . Accepted: 27 June 2011 . Published Online: 18 July 2011

J. D. Mar, X. L. Xu, J. J. Baumberg, A. C. Irvine, C. Stanley, and D. A. Williams

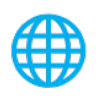

\section{ARTICLES YOU MAY BE INTERESTED IN}

Voltage-controlled electron tunneling from a single self-assembled quantum dot embedded in a two-dimensional-electron-gas-based photovoltaic cell

Journal of Applied Physics 110, 053110 (2011); https://doi.org/10.1063/1.3633216

Charge state control in single InAs/GaAs quantum dots by external electric and magnetic fields

Applied Physics Letters 105, 041109 (2014); https://doi.org/10.1063/1.4891828

Electrical control of fine-structure splitting in self-assembled quantum dots for entangled photon pair creation

Applied Physics Letters 97, 221108 (2010); https://doi.org/10.1063/1.3522655

\section{Lock-in Amplifiers up to 600 $\mathrm{MHz}$}



\title{
Electrically tunable hole tunnelling from a single self-assembled quantum dot embedded in an $\boldsymbol{n}$ - $\boldsymbol{i}$-Schottky photovoltaic cell
}

\author{
J. D. Mar, ${ }^{1,2, a)}$ X. L. Xu, ${ }^{1}$ J. J. Baumberg, ${ }^{2}$ A. C. Irvine, ${ }^{3}$ C. Stanley,${ }^{4}$ and D. A. Williams ${ }^{1}$ \\ ${ }^{1}$ Hitachi Cambridge Laboratory, Cavendish Laboratory, Cambridge CB3 OHE, United Kingdom \\ ${ }^{2}$ NanoPhotonics Centre, Cavendish Laboratory, University of Cambridge, Cambridge CB3 OHE, \\ United Kingdom \\ ${ }^{3}$ Microelectronics Research Centre, Cavendish Laboratory, University of Cambridge, Cambridge CB3 OHE, \\ United Kingdom \\ ${ }^{4}$ Department of Electronics and Electrical Engineering, University of Glasgow, Glasgow G12 8QQ, \\ United Kingdom
}

(Received 10 June 2011; accepted 27 June 2011; published online 18 July 2011)

\begin{abstract}
We perform excitation-intensity-dependent measurements of the neutral exciton $\left(\mathrm{X}^{0}\right)$ photocurrent (PC) peak amplitude from a single InAs/GaAs self-assembled quantum dot (QD) embedded in the intrinsic region of an $n$ - $i$-Schottky photodiode. Since resonant laser-excitation of the $\mathrm{X}^{0}$ transition cannot occur until the comparatively slow hole tunnels out of the QD, we observe a saturation of the PC peak amplitude towards high excitation-intensities, allowing us to determine the hole tunnelling time by fitting with an appropriate theoretical model. By repeating this measurement for a range of bias voltages, we obtain the hole tunnelling time as a function of vertical electric field, showing that it can be tuned by nearly two orders of magnitude. Finally, we find that the hole tunnelling rate can be described accurately by a theoretical model based on a Wentzel-KramersBrillouin approximation to yield precise values for the QD height and hole confinement potential. (C) 2011 American Institute of Physics. [doi:10.1063/1.3614418]
\end{abstract}

The application of self-assembled quantum dots (QDs) towards next-generation quantum optoelectronic devices has recently been the focus of an intense research effort for the implementation of quantum information processing (QIP), due to their strong three-dimensional quantum confinement of electron-hole $(e-h)$ pairs into discrete energy levels. Taking advantage of the high optical quality of self-assembled QDs, such devices function as interfaces between optical fields and an electronic circuit. Examples of such QD-based optoelectronic devices include charge-tunable QD diodes, ${ }^{1,2}$ electrically pumped single-photon sources, ${ }^{3,4}$ and single-QD photodiodes. ${ }^{5,6}$ In particular, the latter reads-out electrically the population of the optically excited QD excitonic two-level system by the amplitude of a photocurrent (PC) signal, which is the result of $e-h$ pair ionization and tunnelling out from the QD into an electronic circuit. Until now, however, all work on bias-voltage-dependent carrier tunnelling in single-QD photodiodes has concentrated solely on that of electrons. ${ }^{5,7}$

Here, we provide an investigation into bias-voltage-dependent hole tunnelling from a single InAs/GaAs selfassembled QD embedded in an $n-i$-Schottky photodiode, which can operate also as a single-QD photovoltaic cell, by performing excitation-intensity-dependent measurements of the neutral exciton $\left(X^{0}\right)$ PC peak amplitude. Since resonant laser-excitation of the $\mathrm{X}^{0}$ transition is blocked until the comparatively slow hole tunnels out of the QD, we observe a saturation of the $\mathrm{X}^{0} \mathrm{PC}$ peak amplitude $I_{\text {peak }}$ towards high excitation-intensities, permitting us to then extract the hole tunnelling time $\tau_{T}^{h}$ by fitting an appropriate theoretical model to the experimental data. Repeating this measurement for a

\footnotetext{
${ }^{\text {a) }}$ Author to whom correspondence should be addressed. Electronic mail: jm585@cam.ac.uk.
}

range of bias voltages, we determine $\tau_{T}^{h}$ as a function of vertical electric field $F$, demonstrating that it is electrically tunable by nearly two orders of magnitude. Finally, we show that the hole tunnelling rate can be described accurately by a theoretical model based on a one-dimensional (1D) WentzelKramers-Brillouin (WKB) approximation, providing insight into the QD height and hole confinement potential.

As shown in the schematic diagram of Fig. 1(a), the device used in this work was designed for single-QD PC measurements and fabricated as an n-i-Schottky photodiode structure based on a two-dimensional electron gas (2DEG). A single layer of InAs/GaAs self-assembled QDs, which was grown to yield a low surface-density of QDs $\left(\sim 10^{9} \mathrm{~cm}^{-2}\right)$, is embedded in a 250-nm-thick layer of $i$-GaAs and positioned $50 \mathrm{~nm}$ above a Si $\delta$-doped GaAs layer $\left(N_{d}=5 \times 10^{12} \mathrm{~cm}^{-2}\right)$, from which the 2DEG is derived and confined in the resultant V-shaped potential well. ${ }^{8,9}$ In order to perform single-QD PC measurements, submicrometer-sized apertures were etched into an $\mathrm{Al}$ shadow mask via electron-beam lithography to isolate single QDs. In addition, Cr/Au bond pads were formed on the ohmic and Schottky contacts to allow for electrical connection to an external voltage source and current meter. Further details of the device structure are given in Refs. 2 and 5.

Prior to the single-QD PC measurements, we first perform bias-dependent micro-photoluminescence ( $\mu$-PL) spectroscopy in order to promptly locate isolated single QDs, measure their particular $\mathrm{X}^{0}$ transition energy, and determine the bias voltage $V_{b}$ range within which a single-QD PC signal may be expected as a result of $e-h$ pair ionization and tunnelling out from the QD following resonant laser-excitation of the $\mathrm{X}^{0}$ transition [Fig. 1(b)]. ${ }^{2,5}$ With this knowledge, we are then able to obtain an $\mathrm{X}^{0} \mathrm{PC}$ resonance peak from a single QD by sweeping the $\mathrm{X}^{0}$ transition energy through that of a fixed narrow-bandwidth 

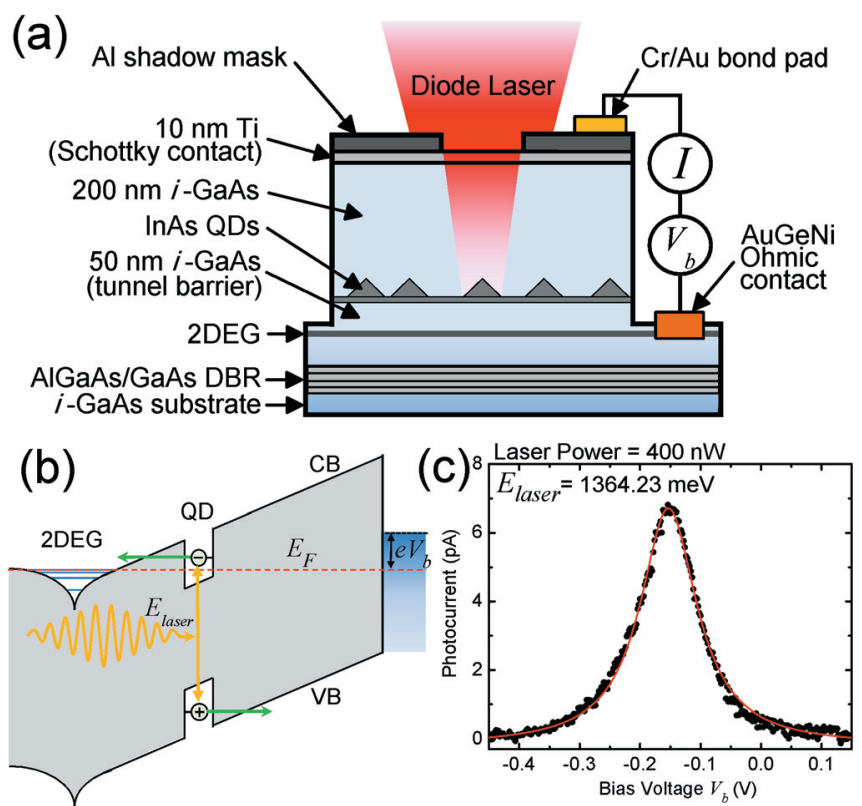

FIG. 1. (Color online) (a) Schematic diagram of the $n$-i-Schottky photodiode used for single-QD PC measurements. (b) Energy-level diagram using resonant laser-excitation. (c) Single-QD X $\mathrm{X}^{0}$ PC peak obtained by sweeping the $\mathrm{X}^{0}$ transition energy through a fixed $E_{\text {laser }}$ via the QCSE. For this PC peak, $E_{\text {laser }}=1364.23 \mathrm{meV}$ and the laser excitation-intensity is $400 \mathrm{nW}$. The solid line is a Lorentzian fit.

cw laser via the quantum-confined Stark effect (QCSE). A typical such PC peak is shown in Fig. 1(c) for one particular QD, on which all measurements in this work will be performed, and for a laser energy $E_{\text {laser }}=1364.23 \mathrm{meV}$ and a laser excitation intensity on the sample of $400 \mathrm{nW}$. The solid line in Fig. 1(c) is a Lorentzian fit curve to the experimental data. Then, using different fixed values of $E_{\text {laser }}$ for such a measurement, the $\mathrm{X}^{0}$ PC resonance peak appears at different $V_{b}$ according to the QCSE. ${ }^{5}$ Note also that the vertical electric field $F$ at the QD layer in our device is defined $\operatorname{as}^{8} F=\left(V_{i}-V_{b}\right) / d$, where $V_{i}$ is the intrinsic built-in potential (Schottky barrier) and $d$ is the distance between the $\mathrm{Si} \delta$-doping and Schottky contact. $V_{i}$ was determined by measuring the value of $V_{b}$ at which the PC signal changed sign (i.e., at the flat-band condition) for non-resonant laser excitation using a $\mathrm{HeNe} \mathrm{cw}$ laser. For all measurements in this work, the sample was maintained at 4.2 $\mathrm{K}$ in a He-flow cold-finger optical cryostat. Detailed descriptions of the $\mu$-PL and PC experimental setups are given in Refs. 2 and 5, respectively.

Due to a renormalization of exciton transition energies as a result of Coulomb interactions in the exciton few-particle states in a QD, the $\mathrm{X}^{0}$ transition cannot be excited resonantly by the laser field until the hole tunnels out of the QD, given that holes tunnel slowest as they possess a much larger effective mass in GaAs compared to electrons. Therefore, a saturation of $I_{\text {peak }}$ is observed towards high excitation-intensities for a given hole tunnelling time, which is dependent inversely on $F$ and reverse bias voltage. Note that, we have correctly taken $I_{\text {peak }}$ (i.e., the PC signal at exact resonance), not any off-resonant $\mathrm{PC}$ signal, as a measure of the $\mathrm{X}^{0}$ absorption for $F$ corresponding to the central $V_{b}$ of a given PC resonance peak. Saturation of $I_{\text {peak }}$ towards high excitation-intensities is demonstrated in Fig. 2, where $I_{\text {peak }}$ is plotted as a function of laser excitation-intensity for $F=35.4 \mathrm{kV} / \mathrm{cm} \quad\left(V_{b}=15.3 \mathrm{mV}\right)$,

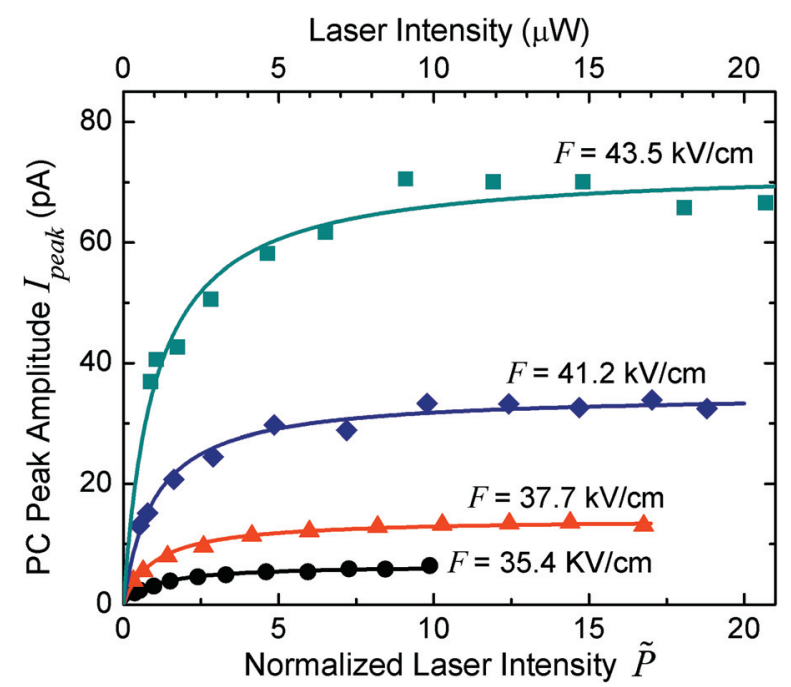

FIG. 2. (Color online) Plot of the PC peak amplitude $I_{\text {peak }}$ as a function of normalized laser excitation-intensity $\tilde{P}$ for $\mathrm{X}^{0}$ resonance peaks centered at several values of $F$. The solid lines are fits using the theoretical model of Eq. (1).

$37.7 \mathrm{kV} / \mathrm{cm}\left(V_{b}=-41.6 \mathrm{mV}\right), 41.2 \mathrm{kV} / \mathrm{cm} \quad\left(V_{b}=-128.9\right.$ $\mathrm{mV})$, and $43.5 \mathrm{kV} / \mathrm{cm}\left(V_{b}=-186.3 \mathrm{mV}\right)$. The increase in the $I_{\text {peak }}$ saturation PC $I_{\text {sat }}$ for increasing $F$ is the result of a decreasing $\tau_{T}^{h}$. This saturation effect of $I_{\text {peak }}$ in our $\mathrm{X}^{0}$ twolevel system can be described by the following theoretical model: $: 5,10$

$$
I_{\text {peak }}=\frac{e}{2 \tau_{T}^{h}} \frac{\tilde{P}}{\tilde{P}+1}=I_{\text {sat }} \frac{\tilde{P}}{\tilde{P}+1},
$$

where $\tilde{P}$ is the normalized laser excitation-intensity on the sample and $e$ is the elementary charge. As we are exciting the $\mathrm{X}^{0}$ transition with a resonant cw laser, note that the factor of $1 / 2$ in $I_{\text {sat }}=e / 2 \tau_{T}^{h}$ is due to the competing processes of absorption and stimulated emission compensating each other under high excitation-intensities, resulting in the population of $\mathrm{X}^{0}$ being $1 / 2$. Fitting the theoretical model of Eq. (1) to the experimental data in Fig. 2 yields $I_{\text {sat }}=6.54 \pm 0.09,14.2$ $\pm 0.1,35.0 \pm 0.4,73 \pm 1 \mathrm{pA}$ and thus $\tau_{T}^{h}=12.3 \pm 0.2,5.64$ $\pm 0.05,2.29 \pm 0.03,1.10 \pm 0.02 \mathrm{~ns}$ for $F=35.4,37.7,41.2$, $43.5 \mathrm{kV} / \mathrm{cm}$, respectively, given that the laser excitation-intensity on the sample was normalized to $1 \mu \mathrm{W}$ in the plot's bottom $x$-axis in order to perform an accurate fit.

We now repeat the above measurement to obtain $\tau_{T}^{h}$ for a series of values of $V_{b}$ throughout the bias range within which a PC peak can be measured in our device, thereby producing experimental data for $\tau_{T}^{h}$ as a function of $F$. The results of such a series of measurements are presented in Fig. 3, showing $\tau_{T}^{h}$ (circles) and the corresponding $I_{\text {sat }}$ (squares) as a function of $F$ and its corresponding $V_{b}$. Notice that $\tau_{T}^{h}$ can be tuned by nearly two orders of magnitude and, specifically, from $13.6 \mathrm{~ns}(F=34.9 \mathrm{kV} / \mathrm{cm})$ down to $175 \mathrm{ps}$ $(F=54.2 \mathrm{kV} / \mathrm{cm})$ over the $V_{b}$ range for a measurable PC peak. Additionally, it is worth noting that, as shown in Fig. 3 and also previously by us in Ref. 5, we observed a PC signal from the QD at zero-bias-voltage conditions (i.e., $V_{b}=0 \mathrm{~V}$ ), thereby confirming that our device is capable of operating also as a QD-based photovoltaic cell. The hole tunnelling rate $R_{T}^{h}$ out of the QD can be modeled via a 1D (along the growth direction) WKB approximation by ${ }^{2,5,11}$ 


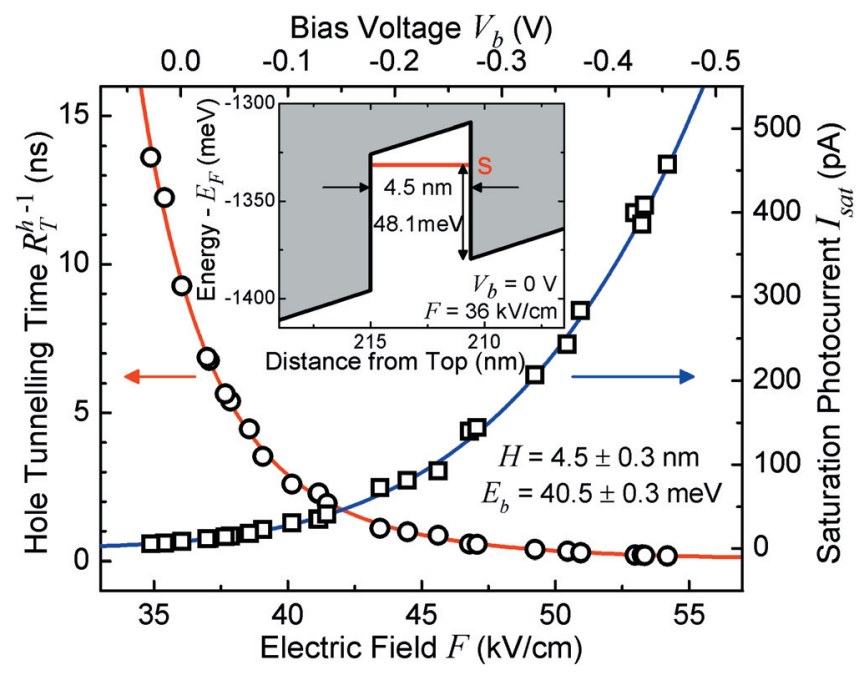

FIG. 3. (Color online) Plot of $I_{\text {sat }}$ (squares) and hole tunnelling time $R_{T}^{-1}$ (circles) as a function of $F$. The solid lines are fits using the theoretical model based on a 1D WKB approximation in Eq. (2), yielding $H=4.5 \pm$ $0.3 \mathrm{~nm}$ and $E_{b}=40.5 \pm 0.3 \mathrm{meV}$. Inset: Theoretical calculation of the valence band and QD $s$-shell hole eigenvalue using a 1D self-consistent Poisson-Schrödinger solver.

$$
R_{T}^{h}=\frac{\hbar \pi}{2 m_{h}^{*} H^{2}} \exp \left[\frac{-4}{3 \hbar e F} \sqrt{2 m_{h}^{*} E_{b}^{3}}\right],
$$

where $m_{h}^{*}=0.59 m_{e}$ (Ref. 12) is the heavy-hole effective mass in GaAs along the growth direction and $m_{e}$ is the electron mass in vacuum, $H$ is the QD height, and $E_{b}$ is the tunnel barrier height for the hole. As can be seen from the solid line in Fig. 3, the theoretical model of Eq. (2) agrees remarkably well with the experimental data throughout the measured range of $F$. Therefore, as shown in the figure, we are able to extract precise values for $H$ and $E_{b}$ as a result of the model fitting. To support the accuracy of the model of Eq. (2), we point out that the value of $H$ obtained here agrees very well with that obtained through our previous work ${ }^{5}$ on $F$-dependent electron tunnelling rates using the same QD. We also wish to emphasize that, while a similar model has been applied to electron tunnelling rates, ${ }^{2,5,7}$ the theoretical model of Eq. (2) has not been used previously to describe electric-field-dependent hole tunnelling rates from a single QD. Finally, it is worth noting that, since the measurements in this work were performed at low temperatures $(\sim 4.2 \mathrm{~K})$, carrier escape from the QD is the result of direct tunnelling from a bound QD $s$-shell state to the GaAs conduction or valence band through the triangular potential barrier, rather than the result of thermionic emission or thermally assisted tunnelling via the InAs wetting layer (WL) states, ${ }^{13}$ which are higher in energy relative to those of the QD $s$-shell due to the stronger quantum confinement along the growth direction in the $2 \mathrm{D}$ WL. Therefore, we take $E_{b}$ in Eq. (2) to be the height of the GaAs triangular tunnel barrier for holes tunnelling directly out of the QD.

The inset of Fig. 3 shows theoretical calculations of the valence band and the QD $s$-shell hole eigenstate using a 1D self-consistent Poisson-Schrödinger solver. By performing these calculations in 1D, we have assumed that carrier tunnelling occurs only along the growth (vertical) direction due to the large vertical electric field and that the carrier wave- functions in the QD are positioned centrally in the plane of a truncated pyramidal QD. Using $H=4.5 \mathrm{~nm}$ and an $\mathrm{X}^{0}$ transition energy of $1364 \mathrm{meV}$, which corresponds to the $\mathrm{X}^{0}$ transition energy of the measured $\mathrm{QD}$, the results of the calculation yield a tunnel barrier height of $48.1 \mathrm{meV}$ for the QD $s$-shell hole. This value agrees reasonably well with the value of $E_{b}$ obtained from the fit shown in the main part of Fig. $3\left(E_{b}=40.5 \pm 0.3 \mathrm{meV}\right)$, thereby further supporting the accuracy of the theoretical model for $R_{T}^{h}$ in Eq. (2).

In this letter, we have demonstrated electrically tunable hole tunnelling from a single self-assembled QD embedded in an $n-i$-Schottky photovoltaic cell by conducting excitation-intensity-dependent measurements of the $\mathrm{X}^{0} \mathrm{PC}$ peak amplitude. A saturation of the $\mathrm{X}^{0} \mathrm{PC}$ peak amplitude was observed towards high excitation-intensities, allowing us to derive $\tau_{T}^{h}$ through fitting with an appropriate theoretical model. Performing this measurement for a range of $V_{b}$, we obtained $\tau_{T}^{h}$ as a function of $F$, showing that it is tunable by nearly two orders of magnitude. Finally, we confirmed that $R_{T}^{h}$ can be described accurately by a theoretical model based on a 1D WKB approximation to generate precise values for $H$ and $E_{b}$. Our comprehensive investigation into $F$-dependent hole tunnelling should have applications in the design of QD-based diode devices. Such devices may include hole charge-tunable QD diodes and single-QD photodiodes for the implementation of hole-spin-based QIP, while the latter may benefit from the observed large tunability of $\tau_{T}^{h}$ to perform initialization, storage, and readout of a hole spin. ${ }^{14,15}$ Additionally, the results of our investigation into hole tunnelling rates may also provide insight towards the design of efficient QD-based photodetectors and photovoltaic cells for solar energy.

J.D.M. gratefully acknowledges financial support from HEFCE, CCT, and NSERC (PGS M, PGS D).

${ }^{1}$ R. J. Warburton, C. Schäflein, D. Haft, F. Bickel, A. Lorke, K. Karrai, J. M. Garcia, W. Schoenfeld, and P. M. Petroff, Nature 405, 926 (2000).

${ }^{2}$ J. D. Mar, X. L. Xu, J. J. Baumberg, F. S. F. Brossard, A. C. Irvine, C. Stanley, and D. A. Williams, Phys. Rev. B 83, 075306 (2011).

${ }^{3}$ X. L. Xu, D. A. Williams, and J. R. A. Cleaver, Appl. Phys. Lett. 85, 3238 (2004).

${ }^{4}$ Z. Yuan, B. E. Kardynal, R. M. Stevenson, A. J. Shields, C. J. Lobo, K. Cooper, N. S. Beattie, D. A. Ritchie, and M. Pepper, Science 295, 102 (2002).

${ }^{5}$ J. D. Mar, X. L. Xu, J. J. Baumberg, A. C. Irvine, C. Stanley, and D. A. Williams, e-print arXiv:1106.1422v1 [cond-mat.mes-hall].

${ }^{6}$ F. Findeis, M. Baier, E. Beham, A. Zrenner, and G. Abstreiter, Appl. Phys. Lett. 78, 2958 (2001).

${ }^{7}$ R. Oulton, J. J. Finley, A. D. Ashmore, I. S. Gregory, D. J. Mowbray, M. S. Skolnick, M. J. Steer, S.-L. Liew, M. A. Migliorato, and A. J. Cullis, Phys. Rev. B 66, 045313 (2002).

${ }^{8}$ K. Ploog, J. Cryst. Growth 81, 304 (1987).

${ }^{9}$ L. M. R. Scolfaro, R. P. Camata, J. M. V. Martins, and J. R. Leite, Superlattices Microstruct. 12, 203 (1992).

${ }^{10}$ E. Beham, A. Zrenner, F. Findeis, M. Bichler, and G. Abstreiter, Appl. Phys. Lett. 79, 2808 (2001).

${ }^{11}$ J. D. Mar, X. L. Xu, J. S. Sandhu, A. C. Irvine, M. Hopkinson, and D. A. Williams, Appl. Phys. Lett. 97, 221108 (2010).

${ }^{12}$ M. A. Cusack, P. R. Briddon, and M. Jaros, Phys. Rev. B 56, 4047 (1997).

${ }^{13}$ W.-H. Chang, T. M. Hsu, C. C. Huang, S. L. Hsu, C. Y. Lai, N. T. Yeh, T. E. Nee, and J.-I. Chyi, Phys. Rev. B 62, 6959 (2000).

${ }^{14}$ D. Heiss, S. Schaeck, H. Huebl, M. Bichler, G. Abstreiter, J. J. Finley, D. V. Bulaev, and D. Loss, Phys. Rev. B 76, 241306(R) (2007).

${ }^{15}$ A. J. Ramsay, S. J. Boyle, R. S. Kolodka, J. B. B. Oliveira, J. Skiba-Szymanska, H. Y. Liu, M. Hopkinson, A. M. Fox, and M. S. Skolnick, Phys. Rev. Lett. 100, 197401 (2008). 